\title{
Outcome for acute bronchitis, bronchiolitis, and pneumonia in infancy
}

\author{
J Y Q MOK AND H SIMPSON
}

The Royal Hospital for Sick Children, Edinburgh and the Department of Child Health, University of Leicester

SUMMARY The clinical and respiratory function characteristics of 200 children 7 years after their admission to hospital with acute lower respiratory tract infection in infancy have been presented. ${ }^{1}$ Results were subsequently analysed according to disease category (bronchitis, bronchiolitis, or pneumonia) at initial presentation. Within each diagnostic category recurrent cough and wheeze, a tendency for colds 'to go to the chest', medication, absence from school, and family doctor consultations were significantly increased. Ventilatory function was diminished and bronchial reactivity increased when compared with matched controls. Studies of a different design are required to elucidate the mechanisms whereby symptoms are increased, ventilatory function impaired, and bronchial reactivity increased after severe lower respiratory infection in infancy.

We have previously reported preliminary findings of a case control, follow up study of 200 children admitted to hospital with acute lower respiratory tract infection in infancy. ${ }^{1}$ When reviewed after 7 years, index children had an increased prevalence of cough, wheeze, and established asthma; ventilatory function was impaired and bronchial reactivity increased compared with matched controls. The atopic backgrounds of index and control children were similar but the former were at a considerable disadvantage with regard to social and family characteristics.

We have examined additional factors that may have influenced outcome, and report here the effects of index diagnosis (bronchitis, bronchiolitis, and pneumonia) on subsequent respiratory status. Our accompanying paper ${ }^{2}$ discusses the occurrence of recurrent or persistent cough and wheeze (irrespective of clinical diagnosis) and the possible influence of atopic status and bronchial reactivity on prognosis.

\section{Subjects and methods}

Details of index children and controls, criteria for their selection, the plan of the investigation undertaken, and a description of our methods were given in the preliminary report. ${ }^{1} \mathrm{~A}$ brief summary is given here.
Subjects. The 200 index children were selected at random from a larger group of infants admitted to hospital with acute lower respiratory tract infection in infancy. In 100 children, respiratory syncytial virus infection was proved; in the remaining 100 , infection was not confirmed by culture or immunofluorescent investigation of nasopharyngeal secretions. Each control child was chosen from the same class in the same school as the index child, and matched for sex, age and, where possible, height. None of the control children had had a respiratory illness in infancy necessitating admission to hospital.

Methods. Index children had carefully documented clinical records, including chest radiographs, and diagnoses were coded according to the criteria suggested by Court $^{3}$ as a prelude to a multicentre study that was subsequently published. ${ }^{4}$ All records were reviewed by one person (JM) to ensure that Court's criteria had been adhered to. Thus, several cases of 'bronchiolitis' were reclassified as 'pneumonia', before inclusion in the study, largely on the basis of chest radiological findings.

Index and control children attended with their parents for review about 7 years later. A detailed questionnaire on clinical, social, and family aspects of health since the index illness was completed, followed by clinical examination and tests of respiratory function. General practitioner records were 
scrutinised in over two thirds of cases, including all index and control children reporting subsequent cough or wheeze.

\section{Statistical analysis}

For matched pairs, continuous data were analysed using Student's $t$ test and the Wilcoxon test for non-parametric data. Analysis of categorical or qualitative data was performed using McNemar's test. $^{5}$

\section{Results}

Diagnostic category. The index illness was bronchitis in 45 children, bronchiolitis in 104, and pneumonia in 51. The boy:girl ratio was almost $2: 1$ for bronchitis and bronchiolitis and 1:1 for pneumonia. Within each subgroup, index children were younger by 0.2 years on average $(P<0.001)$, and smaller by approximately $2 \mathrm{~cm}(\mathrm{P}<0.001-\mathrm{P}<0.02)$ than corresponding control children. Children who had had pneumonia were lighter at birth than their controls (mean (SD), index $3 \cdot 1(0 \cdot 7) \mathrm{kg}$, controls $3.3(0 \cdot 4)$ $\mathrm{kg} ; \mathrm{P}<0.03)$ and fewer index than control children in the bronchiolitis subgroup were breast fed (index $8.7 \%$, controls $21.2 \%$; $\mathrm{P}<0.02$ ).

Table 1 gives details of subsequent outcome. 'Established' respiratory disorders were diagnosed on the basis of clinical findings at follow up, scrutiny of general practitioner records, and, where applicable, hospital case records. Thus, asthma was defined as four or more episodes of wheeze requiring medical attention in the preceding year, and by the same criteria, bronchitis as four or more episodes of cough.

Respiratory symptoms were more common in index subgroups than in their respective case control groups. Cough or wheeze was greatest in the year or two after index illnesses, and gradually decreased thereafter. An increase in asthma and bronchitis was observed at the age of 7 years in each subgroup, but did not reach statistical significance in any. For each diagnostic subgroup, index children lost more schooling, consulted their general practitioners more often, and received more medication (mainly antibiotics) than their respective case controls.

Children with bronchitis did not differ from controls with respect to social class, position in family, number of siblings, parental smoking habits, or age of parents at follow up. More children with bronchiolitis and pneumonia came from social classes IV and V ( $P$ values $<0.05)$, and fewer children with those conditions were first born (respective $\mathbf{P}$ values $<0.01$ and $<0.05$ ). The number of siblings of children who had had pneumonia was significantly increased $(P<0.001)$.

When atopic backgrounds were assessed, no differences were seen between index and control children in the personal histories of eczema, hayfever, or food allergy, nor did differences exist between the three subgroups. The family histories were also similar, with the exception that asthma was reported more frequently among first degree relatives of children with bronchitis (index $60 \%$, control $28.9 \%, \mathrm{P}<0.001$ ).

Table 2 gives the results of respiratory function tests, each expressed as a percentage of the pre-

Table 1 Respiratory status at 7 years of age, in relation to initial clinical diagnosis

\begin{tabular}{|c|c|c|c|c|c|c|}
\hline & \multicolumn{2}{|l|}{$\begin{array}{l}\text { Bronchitis } \\
(n=45)\end{array}$} & \multicolumn{2}{|c|}{$\begin{array}{l}\text { Bronchiolitis } \\
(n=104)\end{array}$} & \multicolumn{2}{|l|}{$\begin{array}{l}\text { Pneumonia } \\
(n=51)\end{array}$} \\
\hline & Index & Control & Index & Control & Index & Control \\
\hline \multicolumn{7}{|l|}{ Cough $(\%)$} \\
\hline At any time & $42 \cdot 2^{\circ}$ & $11 \cdot 1$ & $29 \cdot 8^{\dagger}$ & $14 \cdot 4$ & $37 \cdot 3^{+}$ & $11 \cdot 8$ \\
\hline In past year & $15 \cdot 6^{+}$ & 0 & $15 \cdot 4$ & 7.7 & $21 \cdot 6$ & $5 \cdot 9$ \\
\hline Colds 'going to chest' $(\%)$ & $60 \cdot\left(0^{\ddagger}\right.$ & $17 \cdot 8$ & $51 \cdot 9^{\ddagger}$ & $19 \cdot 2$ & $47 \cdot 1^{\ddagger}$ & $25 \cdot 5$ \\
\hline \multicolumn{7}{|l|}{ Tendency to wheeze $(\%)$} \\
\hline At any time & $60 \cdot 0^{\ddagger}$ & 8.9 & $44 \cdot 2^{\ddagger}$ & $18 \cdot 3$ & $41 \cdot 2^{\ddagger}$ & $21 \cdot 6$ \\
\hline In past year & $6 \cdot 7$ & $2 \cdot 2$ & $10 \cdot 6$ & 0 & $13 \cdot 7^{\circ}$ & $2 \cdot 0$ \\
\hline Medication in past year $(\%)$ & $42 \cdot 2^{+}$ & $15 \cdot 6$ & $46 \cdot 2^{\ddagger}$ & $15 \cdot 4$ & $47 \cdot 1^{\ddagger}$ & $23 \cdot 5$ \\
\hline Antibiotics & $37 \cdot 8$ & $13 \cdot 3$ & $42 \cdot 3$ & $14 \cdot 4$ & $43 \cdot 1$ & $19 \cdot 6$ \\
\hline Bronchodilators & $11 \cdot 1$ & $4 \cdot 4$ & $10 \cdot 6$ & $4 \cdot 8$ & $11 \cdot 8$ & 3.9 \\
\hline $\begin{array}{l}\text { Weeks off school for respiratory illness in past year } \\
(\text { mean }(S D))^{\$}\end{array}$ & $1 \cdot 4(2 \cdot 2)$ & $0.7(1.0)$ & $1.8(2.6)^{\circ}$ & $0.8(1.5)$ & $2 \cdot 4(6 \cdot 0)^{*}$ & $0.8(1.1)$ \\
\hline Established asthma (\%) & $13 \cdot 3$ & $2 \cdot 2$ & 6.7 & $1 \cdot 0$ & $7 \cdot 8$ & 5.9 \\
\hline Established bronchitis (\%) & 0 & 0 & $4 \cdot 8$ & 0 & 3.9 & $2 \cdot()$ \\
\hline
\end{tabular}

${ }_{\mathrm{P}}^{\mathrm{P}}<0 \cdot 01 ;{ }^{\dagger} \mathrm{P}<0 \cdot 05 ;{ }^{\ddagger} \mathrm{P}<0 \cdot\left(001\right.$. Statistical analysis, McNemar`s test; ${ }^{\S}$ Wilcoxon test. 
Table 2 Respiratory function tests in relation to initial diagnosis

\begin{tabular}{|c|c|c|c|c|c|c|}
\hline & \multicolumn{2}{|c|}{$\begin{array}{l}\text { Bronchitis } \\
(n=44)\end{array}$} & \multicolumn{2}{|c|}{$\begin{array}{l}\text { Bronchiolitis } \\
(n=102)\end{array}$} & \multicolumn{2}{|c|}{$\begin{array}{l}\text { Pneumonia } \\
(n=51)\end{array}$} \\
\hline & Index & Control & Index & Control & Index & Control \\
\hline FEV in $0.75 \sec (\%$ predicted $)$ & $89 \cdot 2$ & $91 \cdot 2$ & $87 \cdot 0^{\circ}$ & $92 \cdot 6$ & $91 \cdot 5$ & $94 \cdot 6$ \\
\hline FEV in $1.0 \mathrm{sec}(\%$ predicted $)$ & $90 \cdot 4$ & $94 \cdot 5$ & $90 \cdot 7^{\dagger}$ & $94 \cdot 8$ & $93 \cdot 3$ & 95.7 \\
\hline FVC ( $\%$ predicted $)$ & 8.3 .7 & $86 \cdot 6$ & $86 \cdot 2$ & $86 \cdot 4$ & 88.7 & 88.6 \\
\hline $\mathrm{FEF}_{25-75}$ (\% predicted) & $96 \cdot 1$ & $99 \cdot 1$ & $89 \cdot 1^{\circ}$ & $101 \cdot 3$ & $93 \cdot 4^{\dagger}$ & $107 \cdot 2$ \\
\hline FEV:FVC & 0.90 & 0.91 & $0.88^{*}$ & 0.91 & $0 \cdot 89$ & 0.91 \\
\hline Fall in PEFR of $10 \%$ after exercise $(\%)^{\ddagger}$ & $37 \cdot 8$ & $20 \cdot 0$ & $32 \cdot 7$ & $25 \cdot 0$ & $35 \cdot 3$ & $21 \cdot 6$ \\
\hline
\end{tabular}

FEV = forced expiratory volume $; \mathrm{FVC}=$ forced vital capacity; $\mathrm{FEF}_{25-75}=$ forced mid-expiratory flow (between $25 \%$ and $75 \%$ of $\mathrm{FVC}$ ); PEFR = peak expiratory flow rate.

P $<00.01 ;{ }^{\dagger} \mathrm{P}<0 \cdot 05$. Statistical analysis, Student's paired $t$ test; ${ }^{\ddagger}$ McNemar’s test.

dicted normal value for height. Children with bronchiolitis had a significant reduction in forced expiratory volume in one and in three quarters of a second, forced mid-expiratory flow (between $25 \%$ and $75 \%$ of forced vital capacity), and in forced expiratory volume in one second:forced vital capacity. Ventilatory function was also lower in bronchitis and pneumonia index children, but this did not reach statistical significance. Bronchial reactivity was increased in each index subgroup, but not significantly. As the numerical differences between index subgroups might have affected the results obtained, an analysis of variance was performed between index children and controls for each respiratory function variable measured. This showed that the differences observed within a disease category exceeded the differences between disease categories. We cannot, therefore, conclude that bronchiolitis confers specific risk with regard to ventilatory function, in view of the relatively smaller number of children studied with a history of bronchitis or pneumonia.

\section{Discussion}

These results suggest that the outcome of acute lower respiratory tract infections in infancy is unaffected by the type of index illness suffered. Previous workers have concentrated mainly on the sequelae after acute bronchiolitis; $;^{6-8}$ others have attempted to differentiate acute infectious bronchiolitis from sporadic bronchiolitis that may represent a first asthmatic attack. ${ }^{8-10}$ We had shown no differences in clinical characteristics and outcome between children with proved respiratory syncytial virus infection and those without, ${ }^{1}$ but recognise that this virus could have been responsible for the illness in the latter group despite the lack of virological confirmation.

To our knowledge there has been no previous study relating outcome to the type of index illness suffered. Thus sequelae after bronchitis or pneumonia are less well documented than for bronchiolitis. Respiratory symptoms were increased in our index children, irrespective of the initial clinical diagnosis. Although ventilatory function was unequivocally reduced in children who suffered from bronchiolitis, a diminution in respiratory function was also observed in children who had had bronchitis or pneumonia. Failure to achieve statistical significance may have been caused by the relatively smaller number studied with the latter index conditions.

It may be argued that separation of children on diagnostic criteria based purely on clinical and radiological assessments is artifical, in view of the considerable aetiological overlap between the subgroups defined. There are also difficulties in distinguishing the infant presenting with bronchiolitis from one suffering his first asthmatic attack, as viral respiratory infections are well known to precipitate attacks of asthma. ${ }^{11}$ In assigning index children to a diagnostic grouping Court's recommendations were followed strictly. Moreover, the pneumonia group was not weighted by infants with proved bacterial infections-in each subgroup fewer than $14 \%$ of infants studied had bacterial isolates from throat and nasal swabs, blood culture, or bacteriological studies of tracheal aspirates, on the few occasions when the latter were obtained. On the basis of these findings it seems reasonable to postulate that serious infections of the lower respiratory tract during a 'vulnerable' period of lung growth (whatever the clinical diagnosis) may have contributed directly or indirectly to the subsequent increase in symptoms and impairment of lung function.

We conclude, therefore, that the outcome for acute lower respiratory tract infection severe enough to necessitate admission to hospital in the first year of life is not affected appreciably by the type of index illness suffered. A larger series is 
necessary to settle whether bronchiolitis is more likely than bronchitis or pneumonia to be associated with abnormalities of ventilatory function in later childhood.

This study was supported by a generous grant from the Chest, Heart, and Stroke Association. We thank Dr J M Inglis, Dr M Fulton, Miss C Jagger, and Mrs P Walker. We also thank general practitioners for their cooperation, and all the children and parents who participated in the study.

\section{References}

${ }^{1}$ Mok JYQ, Simpson H. Outcome of acute lower respiratory tract infection in infants: preliminary report of seven year follow-up study. $\mathrm{Br}$ Med J 1982;285:333-7.

2 Mok JYQ, Simpson H. Symptoms, atopy, and bronchial reactivity after lower respiratory infection in infancy. Arch Dis Child 1984;59:299-305.

${ }^{3}$ Court SDM. The definition of acute respiratory illnesses in children. Postgrad Med J 1973;49:771-6.

${ }^{4}$ Clarke SKR, Gardner PS, Poole PM, et al. Respiratory syncytial virus infection: admissions to hospital in industrial, urban and rural areas. Report to the Medical Research Council subcommittee on respiratory syncytial virus vaccines. $\mathrm{Br} \mathrm{Med} \mathrm{J}$ 1978;ii:796-8.
${ }^{5}$ McNemar Q. Note on sampling error of the differences between correlated proportions or percentages. Psychometrika 1947;12:153-7.

${ }^{6}$ Rooney JC, Williams HE. The relationship between proved viral bronchiolitis and subsequent wheezing. $J$ Pediatr 1971;79:774-7.

7 Sims DG, Downham MAPS, Gardner PS, et al. Study of 8 year-old children with a history of respiratory syncytial virus bronchiolitis in infancy. $\mathrm{Br}$ Med $J$ 1978;i:11-4.

${ }^{8}$ Pullan CR, Hey EN. Wheezing, asthma, and pulmonary dysfunction 10 years after infection with respiratory syncytial virus in infancy. $\mathrm{Br}$ Med $J$ 1982;284:1665-9.

${ }^{9}$ Simon G, Jordan WS. Infectious and allergic aspects of bronchiolitis. J Pediatr 1967;70:533-8.

${ }^{10}$ Polmar SH, Robinson LD, Minnefor AB. Immunoglobulin E in bronchiolitis. Pediatrics 1972;50:279-84.

"Berkovitch S, Millian SJ, Snyder RD. The association of viral and mycoplasma infections in the recurrence of wheezing in the asthmatic child. Ann Allergy 1970;28:43-9.

Correspondence to Professor H Simpson, University of Leicester, Department of Child Health, Clinical Sciences Building, Leicester Royal Infirmary, Leicester LE2 7LX.

Received 28 December 1983 